\title{
Laparoscopic management of uterine tumors resembling ovarian sex cord tumors- Pregnancy, recurrence after fertility sparing surgery and literature review
}

\author{
Murat Api ${ }^{1}$ and Yasemin Ceylan ${ }^{2}$ \\ Professor in Obstetrics and Gynecology, University of Health Sciences, Department of Gynecological Oncology ${ }^{1}$ \\ Student at Istanbul Medipol University²
}

Introduction: Uterine tumors resembling ovarian sex cord tumors (UTROSCT) belongs to a rare group of uterine neoplasms. Although, a limited number of malignant and/or recurrent cases have been reported, UTROSCT is generally described as a tumor with benign behaviour. Since the reported cases of UTROSCT are limited and biologic pattern of the tumor has not been clearly elucidated, there is no consensus in the management of the patients. There are only few cases who have undergone fertility sparing surgery and proceeded with successful pregnancies. The objective of the present report is to review the literature relevant to the case presented herein who was first conservatively managed by laparoscopic UTROSCT resection; then consecutively achieved a spontaneous pregnancy and experienced recurrence which was managed by laparoscopic hysterectomy and bilateral salpingectomy.

Materials and Methods: PubMed was searched with the keyword "Uterine tumors resembling ovarian sex cord tumors" from 1976 to 2018. We have found 62 related articles out of 116; from which 128 relevant cases of UTROSCT were analysed.

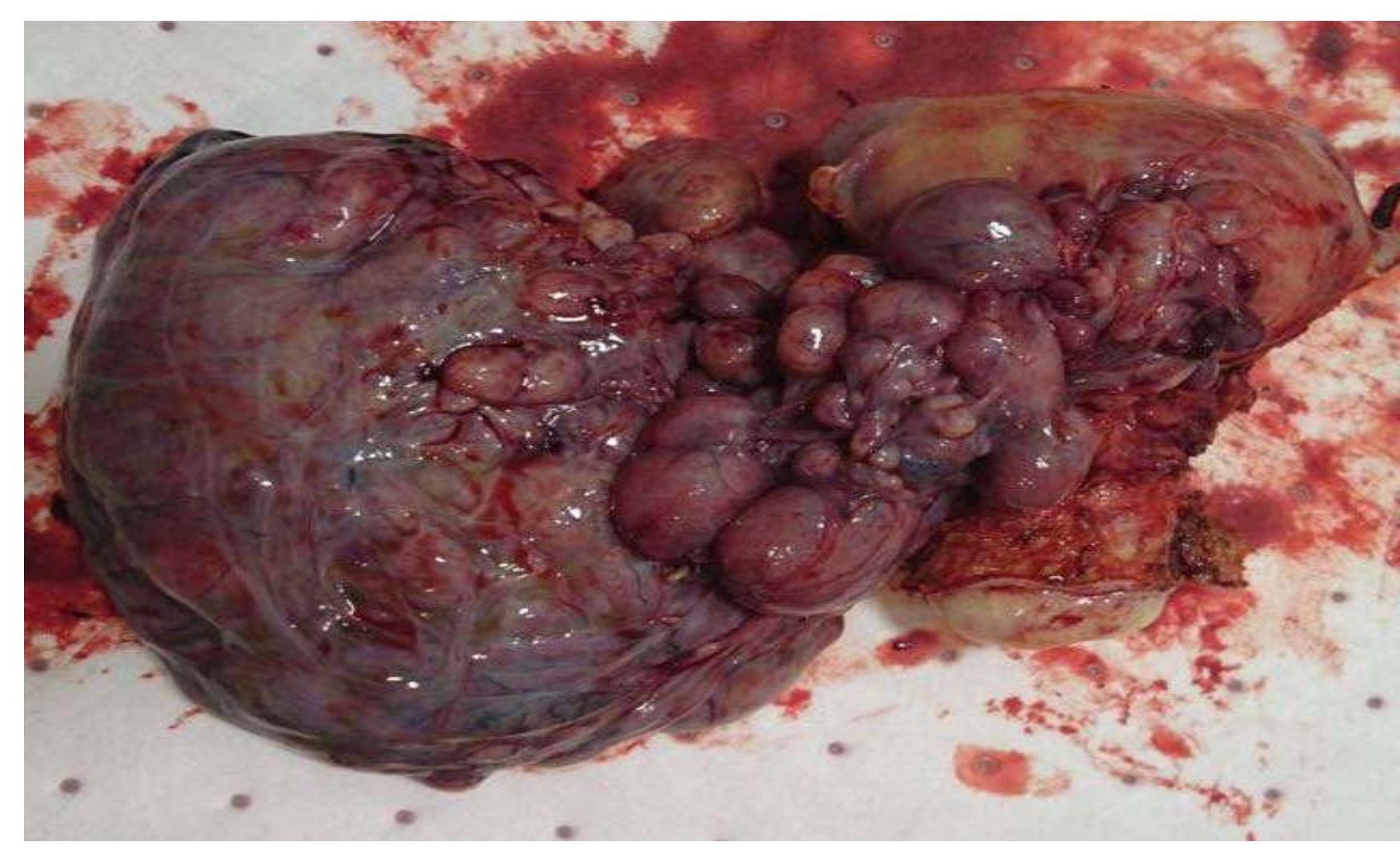

Macroscopic appearance of the recurrent UTROSCT

\begin{tabular}{|c|c|c|c|c|c|}
\hline & Case \# & Age & $\begin{array}{l}\text { Size } \\
(\mathrm{cm})\end{array}$ & Treatment & $\begin{array}{l}\text { Recurrence/ } \\
\text { Metastasis/ } \\
\text { Pregnancy }\end{array}$ \\
\hline $\begin{array}{l}\text { Kuruvila et al. } \\
(2003)\end{array}$ & 1 & 50 & NA & Polypectomy & - \\
\hline $\begin{array}{c}\text { Anastasakis et } \\
\text { al. (2008) }\end{array}$ & 1 & 28 & NA & Hysteroscopic resection & Pregnancy \\
\hline $\begin{array}{l}\text { Nogales et al. } \\
\qquad(2008)\end{array}$ & $1 / 6$ & 76 & NA & Radiotherapy due to morbid obesity & - \\
\hline $\begin{array}{l}\text { Berretta et al. } \\
\quad(2009)\end{array}$ & 1 & 26 & 2.2 & Hysteroscopic resection & - \\
\hline $\begin{array}{l}\text { Garuti et al. } \\
\text { (2009) }\end{array}$ & 1 & 29 & 5 & Hysteroscopic resection & - \\
\hline $\begin{array}{l}\text { Giordano et al. } \\
\quad(2010)\end{array}$ & $1 / 2$ & 26 & 2.2 & Hysteroscopic resection & - \\
\hline $\begin{array}{l}\text { Abdullazade et } \\
\text { al. (2010) }\end{array}$ & $1 / 3$ & 30 & 2 & Myomectomy & - \\
\hline $\begin{array}{l}\text { Jeong et al. } \\
\text { (2015) }\end{array}$ & 1 & 32 & 3.6 & Resectoscopic mass resection, TLH, BS & Pregnancy \\
\hline $\begin{array}{l}\text { Watrowski et } \\
\text { al. (2015) }\end{array}$ & 1 & 22 & 2.2 & Hysteroscopic resection & - \\
\hline $\begin{array}{l}\text { De Franciscis } \\
\text { et al. (2016) }\end{array}$ & 1 & 38 & 1 & Hysteroscopic resection & Pregnancy \\
\hline $\begin{array}{l}\text { Schraag et al. } \\
\quad(2017)\end{array}$ & $2 / 3$ & 24,28 & $\begin{array}{l}1.5 \\
10\end{array}$ & $\begin{array}{l}\text { Laparotomy for resection of the tumor/ } \\
\text { TAH, BS, tumor resection by laparotomy, } \\
\text { removal of both adnexa, part of the vaginal } \\
\text { wall and affected peritoneum }\end{array}$ & $\begin{array}{l}\text { Recurrency/ } \\
\text { Recurrency } \\
\text { after pregnancy }\end{array}$ \\
\hline $\begin{array}{l}\text { Sadeh et al. } \\
\text { (2017) }\end{array}$ & 1 & 57 & 0.9 & Hysteroscopic resection, TLH, BSO & - \\
\hline $\begin{array}{l}\text { Thakur et al. } \\
\text { (2018) }\end{array}$ & 1 & 37 & 1.1 & Laparo-hysteroscopic resection & - \\
\hline $\begin{array}{c}\text { Our Case } \\
(2018)\end{array}$ & 1 & 39 & 15 & Laparoscopic myomectomy, TLH, BS & $\begin{array}{l}\text { Pregnancy and } \\
\text { recurrency }\end{array}$ \\
\hline
\end{tabular}

TABLE 3: Conservatively treated UTROSCT cases:

TAH: Total abdominal hysterectomy, BSO: Bilateral salpingo-oophorectomy BS: Bilateral salpingectomy, TLH: Total laparoscopic hysterectomy

NA: Not available.
TABLE 1: Recurrent UTROSCT cases from the literature review $\mathrm{TAH}$ : Total abdominal hysterectomy

BSO: Bilateral salpingo-oophorectomy, BS: Bilateral salpingectomy PME: Pelvic mass excision, TIR: Tumoral implant resection

TLH: Total laparoscopic hysterectomy NA: Not available.

\begin{tabular}{|c|c|c|c|c|c|}
\hline & $\begin{array}{l}\text { Cas } \\
\text { e\# }\end{array}$ & Age & $\begin{array}{l}\text { Size } \\
(\mathrm{cm})\end{array}$ & Treatment & $\begin{array}{c}\text { Pregnancy } \\
\text { Recurrenc } \\
\text { e }\end{array}$ \\
\hline $\begin{array}{l}\text { Anastasak } \\
\text { is et al. } \\
\text { (2007) }\end{array}$ & 1 & 28 & NA & Hysteroscopic resection & Pregnancy \\
\hline $\begin{array}{l}\text { Jeong et } \\
\text { al. (2015) }\end{array}$ & 1 & 32 & 3.6 & $\begin{array}{c}\text { Hysteroscopic resection, } \\
\text { TLH, BS }\end{array}$ & Pregnancy \\
\hline $\begin{array}{c}\text { De } \\
\text { Franciscis } \\
\text { et al. } \\
(2016)\end{array}$ & 1 & 38 & 1 & Hysteroscopic resection & Pregnancy \\
\hline $\begin{array}{l}\text { Schraag et } \\
\text { al. (2017) }\end{array}$ & $1 / 3$ & 28 & 10 & $\begin{array}{l}\text { Tumor resection by } \\
\text { laparotomy, TAH, BS, } \\
\text { removal of both adnexa, } \\
\text { part of the vaginal wall and } \\
\text { affected peritoneum. } \\
\text { Anastrozole was given } \\
\text { after the recurrency }\end{array}$ & $\begin{array}{c}\text { Recurrency } \\
\text { after } \\
\text { pregnancy }\end{array}$ \\
\hline $\begin{array}{l}\text { Our case } \\
(2018)\end{array}$ & 1 & 39 & 15 & $\begin{array}{c}\text { Laparoscopic } \\
\text { myomectomy, TLH, BS }\end{array}$ & $\begin{array}{l}\text { Recurrenc } \\
\text { e after } \\
\text { pregnancy }\end{array}$ \\
\hline
\end{tabular}

Results: Among the reported 128 cases, $11.7 \%$ of them were conservatively treated initially. Conservative management has been subsequently followed with 5 live births, including our case, whereas 16 cases were diagnosed with recurrence (2 of them following successful pregnancies) who required definitive treatment. For the patients under 40 years of age, fertility preserving treatment ended up with successful pregnancies in $41.6 \%(5 / 12)$ of cases. Our case presented herein is a 39 -year old woman who was initially diagnosed with UTROSCT by hysteroscopic biopsy. To our knowledge, our case is the first to be treated laparoscopically for the recurrence of UTROSCT following laparoscopic fertility sparing surgery proceeding with successful pregnancy.

\begin{tabular}{|c|c|c|c|c|c|}
\hline & $\begin{array}{c}\text { Case } \\
\#\end{array}$ & Age & $\begin{array}{l}\text { Size } \\
(\mathrm{cm})\end{array}$ & Treatment & $\begin{array}{l}\text { Pregnancy/ } \\
\text { Recurrence }\end{array}$ \\
\hline $\begin{array}{l}\text { Anastasakis } \\
\text { et al. (2007) }\end{array}$ & 1 & 28 & NA & Hysteroscopic resection & Pregnancy \\
\hline $\begin{array}{l}\text { Jeong et al. } \\
\quad(2015)\end{array}$ & 1 & 32 & 3.6 & $\begin{array}{c}\text { Hysteroscopic resection, TLH, } \\
\text { BS }\end{array}$ & Pregnancy \\
\hline $\begin{array}{c}\text { De } \\
\text { Franciscis et } \\
\text { al. (2016) }\end{array}$ & 1 & 38 & 1 & Hysteroscopic resection & Pregnancy \\
\hline $\begin{array}{l}\text { Schraag et } \\
\text { al. }(2017)\end{array}$ & $1 / 3$ & 28 & 10 & $\begin{array}{l}\text { Tumor resection by laparotomy, } \\
\text { TAH, BS, removal of both } \\
\text { adnexa, part of the vaginal wall } \\
\text { and affected peritoneum. } \\
\text { Anastrozole was given after the } \\
\text { recurrency }\end{array}$ & $\begin{array}{c}\text { Recurrency } \\
\text { after } \\
\text { pregnancy }\end{array}$ \\
\hline $\begin{array}{c}\text { Our case } \\
(2018)\end{array}$ & 1 & 39 & 15 & $\begin{array}{c}\text { Laparoscopic myomectomy, } \\
\text { TLH, BS }\end{array}$ & $\begin{array}{c}\text { Recurrence } \\
\text { after } \\
\text { pregnancy }\end{array}$ \\
\hline
\end{tabular}

TABLE 2: Pregnancy cases after the treatment of UTROSCT $\mathrm{TAH}$ : Total abdominal hysterectomy, BS: Bilateral salpingectomy TLH: Total laparoscopic hysterectomy NA: Not available.

Conclusion: Fertility sparing surgery via laparoscopic approach seems to be a considerable option of treatment for women with UTROSCT in their reproductive ages. Minimally invasive surgery for UTROSCT is suggested to be applicable in both conservative and definitive management. 